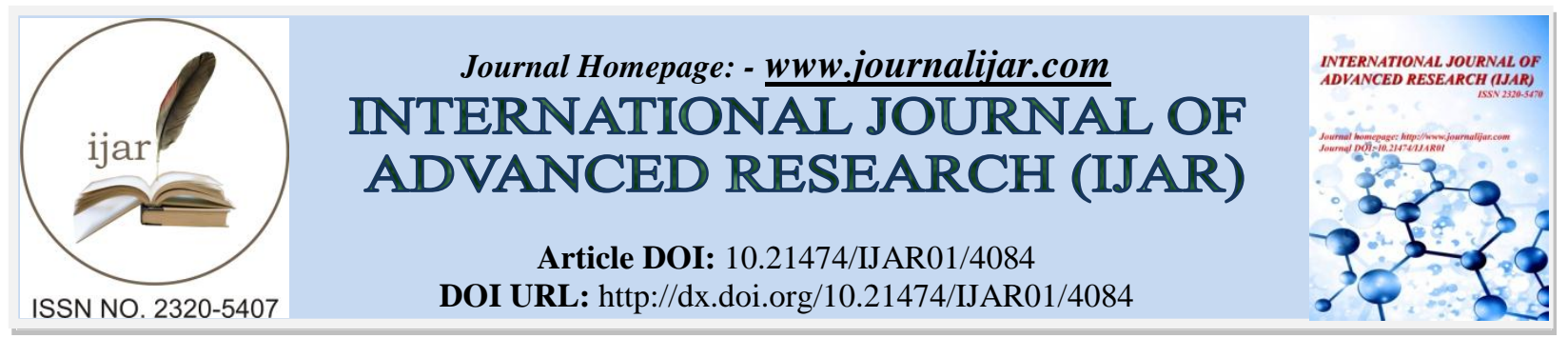

RESEARCH ARTICLE

\title{
CONSANGUINITY AND LIPID PROFILE VARIATIONS IN VARIOUS CLANS OF PAKISTANI POPULATION
}

Iram Anjum ${ }^{1}$ and "Sidra Ajmal' .

1. Department of Biotechnology, Kinnaird College for Women, Lahore, Pakistan.

2. Department of Biochemistry, Kinnaird College for Women, Lahore, Pakistan.

\section{Manuscript Info}

Manuscript History

Received: 06 March 2017

Final Accepted: 01 April 2017

Published: May 2017

Key words:-

Consanguinity, Lipid Profile, Diabetes,

Cardiovascular Diseases, Hypertension,

Chemical Auto-Analyzer.

\begin{abstract}
In Pakistan, consanguinity amongst one's caste is a social norm. Increased rates of cousin marriages give rise to fatal diseases which are inheritable and can pass from one generation to another. In this regard, since no research has been conducted up till now for analyzing the link between consanguinity and castes, the current study aims at estimating the prevalence of consanguinity in Pakistani population in general and in various clans specifically. In addition, to find the relationship of diabetes, hypertension as well as cardiovascular diseases and lipid profile variation with consanguinity was another major objective of this study. The data was collected randomly from a sample size of 400 participants in four different hospitals and lipid profile parameters were evaluated on the basis of chemical auto-analyzer. The results found that Mughals had the highest percentage (43\%) of hypertensive participants while Kashmiri had the highest number of obese participants (14\%). Despite having maximum consanguinity in the Pathan clan (88\%), diabetes and hypertension was more prevalent in Kashmiris while cardiovascular diseases were more prevalent in the Baloch clan. Secondly, Baloch and Gujjars were found to have abnormal values for various parameters (total cholesterol and triglycerides) of lipid profile clearly revealing presence of CVD and earlier onset of CVD among Baloch and Gujjars.
\end{abstract}

Copy Right, IJAR, 2017, All rights reserved.

\section{Introduction:-}

In Pakistan, consanguinity is a social norm and especially in majority of the outskirts and villages where the common practice of cousin marriages is still considerably very constant (Khan et al., 2011). Many scientists have considered consanguineous marriages as being the major risk factors behind various inherited, genetically associated, complex or congenital diseases (Gupta, 1974). Consanguinity basically refers to a marriage between the cousins related biologically. According to the concepts of Sabean (1998), it clearly establishes fixed lines dividing the people from and also connecting the people to others. Furthermore, cousin marriage also binds males and females together in a complex web of in-laws and resultantly helps in providing the base for stronger chances of succession, inheritance, as well as authority.

Consanguinity is witnessed in many societies but specifically in the under-developed and developing countries. For instance, the Gujar along with the Bakerwals living the Jammu and Kashmir strictly follow the tradition of first 
cousin marriages within their own respective community. In addition, they only bring girls from other communities which have high respect or which are greatly recognized in the community but such marriages have failed in getting a reputable image within the entire community. From this case, it becomes very evident that marriage mainly between the parallel cousins as well as cross cousins is greatly preferred because of weaker financial conditions. Moreover, these types of marriages within any specific family group involving reduced rights as well as responsibilities, not involving any extraordinary expenditure on either the feasts or dowry or for exchange of articles demonstrates the fact for why inter-marriages are preferred (Ahmed, 1976).

Both the genetic as well as medical researchers have clearly shown that inter-marriages must be avoided due to the fact that children born after such marriages are greatly affected (Parveen and Rehman, 2012). In this regard, it was said that they have a very high risk of being either physically or mentally retarded or becoming affiliated by adverse defects such as asthma, deafness, blindness, eczema, sickle cell disease, epilepsy, specific cancers along with squint eyes (Temtamy et al., 1998). Hence, it is very important for such families to prepare themselves for accepting the major challenges associated to the prevalence of complex disorders, congenital malformations as well as risk factors because of these marriages which might require getting full and complete information along with comprehensive genetic education and lastly premarital counseling programs for both preventions as well as awareness (Shami, Qaisar and Bittles, 1991).

In relation to consanguinity, previous researches have reported abnormal levels of serum lipids but the overall pattern of cousin marriages in caste/tribal system leading to such abnormality is still unclear (Samatha Venkateswarlu and Siva, 2012). Moreover, abnormal serum lipids have been linked to a chance of developing coronary heart diseases especially in majority of the diabetic patients (World Health Organization, 1994) and is also set as a basic standard of the diabetes care mainly for determination of variation in serum lipid levels in the people suffering from diabetes (Miller, 1999). Both abnormal lipid profiles as well as lipoprotein oxidation (for instance, LDL-C) are now found to be higher in diabetics and are also combined with having a considerably poorer glycaemic control (The American Diabetes Association, 1999).

Hence, the main aim of the paper is to thoroughly describe and analyze various lipid profile parameters among the population of Pakistan and also to identify the number of inter-marriages among the most prevalent clans/tribes of Pakistan. To date, no study has been carried out which compares abnormal values of serum lipids in terms of rate of inter-marriages, thus, there is a dire need for finding out whether such values among the Pakistanis is due to increasing prevalence of consanguinity or not. In this paper, we aim to compare the clans of participants (both male and females) from four different hospitals of Lahore; Punjab Institute of Cardiology, Services, Mayo hospital and Jinnah hospital. In addition, we will also analyze their lipid profile parameters (cholesterol level, total cholesterol, triglycerides, HDL and LDL), Body mass index (BMI) and waist-to-hip (WHR) ratio.

\section{Materials and Methods:- \\ Sample Size:-}

A total of 400 samples of Pakistani population were sampled belonging to ten major castes. These included Rajputs (135/400), Jats (120/400), Syed (45/400), Sheikh and Pathan (25/400 each), Mughal (16/400), Baloch (11/400), Gujjar (10/400), Kashmiri (7/400) and lastly Rehmani (6/400).

\section{Experimental Design:-}

The current study is a randomized study in which data was collected randomly from four different hospitals and participants having origin in different cities of Punjab were included in the study as well. The sampling and data collection was done during February and March 2014. The four different hospitals were Punjab Institute of Cardiology, Services, Mayo hospital and Jinnah hospital. The number of samples collected and tested for lipid profile daily ranged from 20-25. A comprehensive research questionnaire was specifically designed that mainly included all the required information of the study participants.

\section{Anthropometric Measurements:-}

The following anthropometric measurements were recorded for establishing various anthropometric dimensions of the total participants. 


\section{Estimation Of Weight And Height:-}

Weight of the participants was measured with the help of weight machine (Diagnostic-Model No SF-400A) in kilograms $(\mathrm{kg})$. For the height, the height was measured by using standard scale of inches. The participants were requested to remove their shoes and to stand against the wall. After this, their height was carefully marked and was measured by using an inch tape.

\section{Calculation Of BMI:-}

The method of BMI is basically for the measurement of obesity in an individual i.e. total body fat percentage. In this regard, this high body fat level is reported to be linked with a wide range of diseases. In this study, the BMI (body mass index) of the participants was calculated by the following formula i.e. dividing the total body weight of individual $(\mathrm{kg})$ by his/her height $(\mathrm{m} 2)$. BMI calculation formula is:

$$
\mathrm{BMI}=\text { Body Weight }(\mathrm{kg})
$$

Height $\left(\mathrm{m}^{2}\right)$

\section{Estimation Of Blood Pressure:-}

For measuring the blood pressure of participants, the cuff was placed and wrapped around the bare upper left arm about a one inch over the participant's elbow. After this, the disc of the stethoscope was placed over the brachial artery which is located just below the lower edge of the cuff. After this, the cuff was rapidly inflated to reach an exact value of $180 \mathrm{mmHg}$. Once reached, the knob was turned on and the air was allowed to release slowly. At this point, the reading was noted when the very first knocking sound was heard through the stethoscope. This was basically the systolic blood pressure of the participant. For the diastolic pressure, the reading was noted down when no sound of knocking was heard. Standard Value is $120 / 80 \mathrm{mmHg}$.

\section{Laboratory Assay:-}

The four important parameters which were analyzed in every sample were (i) Total-CHOL (ii) Triglycerides (iii) HDL-C and (iv) LDL-C. The serum lipid profile was evaluated with the help of Hitachi 912 Autoanalyzer (model: Roche Diagnostic, Germany).

\section{Collection of Specimen:-}

The participant's blood was collected into red capped vacutainers. The blood was allowed to stand for around 45 min mainly at the room temperature for allowing adequate clotting as well as clot retraction. During this entire clotting period, the blood was left in the sealed tubes. After clotting of the blood, the samples were centrifuged at $1,500 \mathrm{rpm}$ for around $30 \mathrm{~min}$ with a temperature of $4^{\circ} \mathrm{C}$.

\section{Procedure for Estimating Total Cholesterol and Triglyceride:-}

After getting the serum, the reaction conditions were set to normal and were established carefully on the basis of Instrument Settings. An aliquot of $100 \mathrm{ul}$ from the sample was added into the machine's disposable sample cups which were then placed on the carousel of the instrument with the help of using a disposable and sterilized polyethylene transferring pipette. The samples were then arranged on the carousel of instrument. The control samples were also placed into their fixed labeled positions and then the analysis was done electronically. After one hour of completion of the lipid profile test for cholesterol and triglyceride for 200, the results (values) were displayed on the attached computer and were also printed by the Hitachi printer. A separate file was also created for different samples with different labeling. These were carefully assembled into one database after completion of the tests. Same procedure was applied for the next set of 200 samples.

\section{Direct Method for Estimating Amount of HDL and LDL Calculation:-}

Firstly, the direct method for estimating the amount of HDL utilizes sulphated alpha-cyclodextrin mainly in the presence of $\mathrm{Mg}^{+2}$ ions. These form complexes along with the apoB which contains lipoproteins. Followed by this, the polyethylene glycol which is coupled with cholesteryl esterase as well as cholesterol oxidase is used for the measurement of HDL-cholesterol. Absorbance is measured at around $600 \mathrm{~nm}$. Secondly, LDL-cholesterol was basically calculated from the obtained values of total cholesterol along with triglycerides as well as HDL. The following formula was used for calculation of LDL:

$[\mathrm{LDL}-\mathrm{chol}]=[$ total chol $]-[\mathrm{HDL}-\mathrm{chol}]-[\mathrm{TG}] / 5$.

Here $[\mathrm{TG}] / 5$ is an estimate of VLDL-cholesterol and all values are expressed in $\mathrm{mg} / \mathrm{dL}$. 


\section{Statistical Analysis:-}

The data which was obtained from the study was at the end analyzed by using the software Microsoft Excel as well as Statistical Package for Social Sciences (SPSS). Chi square test was used for finding relationship between different parameters.

\section{Results:-}

\section{Distribution of Castes:-}

The study was conducted to analyze variation in body composition parameters (age, body mass indexes, waist-to-hip ratios and blood pressure) and most importantly occurrence of consanguinity and onset complex disorders (diabetes, hypertension and cardiovascular diseases) among the major castes of Pakistan. The castes were collectively divided into ten major groups compromising of sub-castes on the basis of origin and number of participants of this study $(\mathrm{N}=400)$. The maximum number of participants belonged to Rajputs (135/400) followed by Jats (120/400), Syeds (45/400), Sheikhs and Pathans (25/400), Mughals (16/400), Gujjars (11/400), Balochis (10/400), Kashmiris (7/400) and lastly Rehmani (6/400).

\section{Gender Distribution Among Different Castes:-}

The total number of males and females among the castes differed significantly showing male participants to be affected more in terms of diseases than the females. The total percentage of males in Rajput was $81 \%(110 / 135)$ while on the other hand; the total number of females was considerably quite low accounting for only 19\% (25/135). Secondly, the total number of Jat males was $87(72 \%)$ and the female participants included in the study were 33 (28\%). Followed by this, the males in Syed, Sheikh, Pathan and Mughal, accounted for 67\% (30/45), 60\% (15/25), $80 \%$ (20/25), 25\% (4/16) respectively. The females in Syed, Sheikh, Pathan and Mughal accounted for only 33\% (15/45), 40\% (10/25), 20\% (5/25) and 75\% (12/16) respectively. The castes namely Gujjar (11/11), Baloch (10/10), Kashmiri (7/7) and Rehmani (6/6) had 100\% males.

\section{Variation in Body Mass Index Among Different Castes:-}

On the basis of the participant's BMI, the subjects of each caste were divided into four categories as recommended by the WHO for body mass index. The four categories were namely underweight (BMI>18.5), normal (BMI<18.524.9) as well as pre-obese (BMI <25.0-29.9) and lastly the obese (BMI>30.0). maximum number of underweight participants was found in Rehmani accounting for 33\% followed by Gujjar with a percentage of 20\%. Maximum percentage of normal participants were found in the Kashmiris $(71 \%)$ followed by Rajput (70\%). the highest percentage of pre-obese participants was found to be Baloch accounting for $50 \%$ followed by Sheikhs with $44 \%$ and Jat with 35\%. Lastly, the maximum percentage was found to be among the Kashmiris accounting for $14 \%$. They were followed by Sheikh and Mughals with a percentage of $0.04 \%$ each.

\section{Variation of Waist-To-Hip Ratio (WHR) Among Various Castes:-}

The waist-to-hip ratio according to WHO are classified into two categories, the normal and above normal. For men, the normal values should be less than $0.85(<0.85)$ and for females, the ratio should be below $0.75(<0.75)$. For males, maximum number of normal participants belong the Rehmani, Gujjar and Baloch castes accounting for 100\% of total participants. On the other hand, the highest percentage of above normal participants belonged to the Rajputs accounting for $60 \%$ followed by Jat with $24 \%$ of total participants. For the females, maximum number of normal females belonged to the Mughals followed by Sheikh accounting for 58\% and 53\% respectively. On the other hand, maximum number of females having above normal WHR was significantly the Pathans making up $60 \%$ of the entire population followed by Syed with 53\% of total participants.

\section{Variation Of Blood Pressure (Systolic) Among Different Castes:-}

The participants were divided into three categories on the basis of their systolic blood pressure. The three categories were normal blood pressure $(<120)$, prehypertension (120-139) and hypertension $(\geq 140)$. Kashmiri and Rehamni were found to have maximum normal participants while on the other hand, Jats and Mughals have the maximum participants falling in the pre-hypertension category and accounts for $31 \%$ each. Followed by this, Baloch occupies the third position for having maximum number of pre-hypertensive participants. Once again the following figure shows Mughals with the maximum number of hypertensive participants and accounts for $43 \%$. Followed by this, Syed occupies second position with a $28 \%$ percentage of total participants. 


\section{Consanguinity and Diabetes:-}

It was found that Pathans had maximum consanguineous marriages $(88 \%)$ followed by Gujjar (81\%) and Baloch $(81 \%)$. In relation to prevalence of diabetes, then these also show high percentage of diabetic participants however, an important thing to note here were the Kashmiris with maximum proportion of diabetic but comparatively lesser cases of consanguinity (fig I). Secondly, maximum consanguinity was found in Pathan (88\%) but low percentage of family history of diabetes participants. On the other hand, Mughals have the least number of consanguineous marriages but highest percentage of family history for diabetes (fig II). An inverse relationship of consanguinity was found with age of onset of diabetes. All those castes with higher consanguinity have lower age of onset, indicating loss of fitness (fig III).

\section{Consanguinity and Hypertension:-}

Pathans have maximum incidences of consanguinity and highest percentage of hypertensive participants (fig IV). Secondly, highest percentage of family history was found in Pathan, Baloch, Gujjar and Kashmiri (100\% each) and Pathan are found to have maximum cases of hypertension (fig V).

\section{Consanguinity and Cardiovascular Disease:-}

Although maximum consanguinity was found in Pathan (88\%) but maximum cases of CVD were found in the Baloch (89\%) (fig VI). Secondly, maximum family history of CVD was among the Gujjars with second highest percentage of consanguinity (fig VII). There was an inverse relationship between consanguinity and age of onset of cardiovascular disease. The castes practicing more consanguinity have a lower age of onset of this life-threatening disease as well (fig VIII).

\section{Lipid Profile Variations Based On Ethnicity:-}

Baloch (231) were found to have highest values for total cholesterol followed by Pathan (226) (fig IX). Highest triglyceride values were found in Baloch (214) followed by Jat (187) (Fig X). High level of HDL was found among Rehmani (57) and Kashmiri (54) signifying presence of good cholesterol among them (Fig XI). High level of HDL was found among the Pathan females (56) while on the other hand, Rajput, Jat, Syed and Mughal lie in the danger zone (fig XII). High level of LDL was found among the Kashmiris (145) followed by Pathan (145) (fig XIII).

\section{Statistical Analysis:-}

Chi square test was used for analyzing relationship between consanguinity and different parameters. These parameters basically included onset of diseases, family history of disease and lipid profile. According to the Chisquare test, a positive relationship was found mainly between the family history as well as consanguinity. They accounted for $\mathrm{p}$ values being less than 0.05 and had $\mathrm{p}$ values 0.01 and 0.02 respectively. Secondly, it was found that no relationship exists between the consanguinity as well as onset of diabetes. According to the test, strong relationship between consanguinity and family history was found having p-value 0.03 . The results of the test also showed negative relation between consanguinity and family history of CVD. Lastly, the relationship between consanguinity and onset of CVD was not significant.

\section{Discussion:-}

The study was basically conducted to find out the prevalence of three complex disorders along with its relation to lipid profile parameters (total cholesterol, triglyceride, HDL-C and LDL-C), family history of patients and consanguinity in most prevalent castes of Lahore. As compared to the females, the majority of participants being male indicate the fact that males are at more risk of developing cardiovascular diseases which further gives rise to other complications such as diabetes or vice versa and hypertension as well. Each of the ten castes showed majority of male participants and hence clearly pertains to the list of risk factors stated by Mosca et al. (2011). According to them, males are at more risk of developing CVD as compared to the females and this risk can also be increased in presence of other diseases such as diabetes or hypertension which is rendered as a silent killer.

Talking about the body mass index, then according to the World Health Organization, there are four categories into which the individuals can be divided on the basis of their BMI value. The current study showed participants from Rehmani caste falling into the category underweight. In addition, Kashmiris were found to be the normal individuals on the basis of their BMI value but also gained the position of having maximum obese patients. Followed by this, Balochi, Sheikh and Jat had maximum number of pre-obese participants. Considering the BMI of each of these castes as being underweight, normal, pre-obese and obese, it can be assumed according to the findings of Kaplan (1989) that individuals are more susceptible for developing CVD because obesity is more or less linked to onset of 
CVD and other diseases. This was clearly linked to the results of the current study showing Gujjars and Baloch have maximum incidences of CVD and also had maximum participants in pre-obese category if not in obese. However, he also considered BMI as the worst predictor for CVD for diabetes and hypertension due to the fact that other risk factors are more associated to these such as family history, growing age, increased level of cholesterols, sedentary life style, etc.

Waist-to-hip ratio by Koning et al. (2007) is regarded as the best parameter for predicting association between CVD, diabetes and hypertension. This study showed Rajputs, Jat and Kashmiri having maximum number of participants falling above the normal range of WHR and this clearly demonstrates the fact for why they have huge number of individuals suffering from diabetes and hypertension. Talking about the female WHR ratio, then it is also confirmed that above normal values for WHR is a major risk factor for development of CVD and diabetes and this is well demonstrated in the current study.

Increased blood pressure is directly linked to onset of hypertension and ultimately development of CVD. In case of presence of diabetes, the chances of developing hypertensive situation and CVD increases due to the fact that diabetes constrict the capillary walls ultimately giving rise to various heart diseases. Same case was revealed in the current study showing Baloch, Gujjar and Mughals to have maximum participants falling in the pre-hypertensive and hypertensive categories. This can be associated to the study conducted by Kazim et al. (2008) showing increased blood pressure and onset of hypertension. When compared to the consanguinity, it was seen that Baloch, Gujjar, and Kashmiri having maximum percentage of parental consanguinity also had maximum number of participants suffering from hypertension. From this it becomes clear that consanguinity plays an important role in onset of hypertension which may later on give rise to development of CVD.

A positive relationship was found between diabetes and consanguinity and was greatly revealed in case of Rajputs, Sheikhs, Balochs and Gujjars. In addition, although relationship between consanguinity and age of onset for diabetes wasn't found to be significant, it can be said that strong positive family history in Rajputs may trigger earlier onset of disease. Followed by these, Sheikh had the second lowest age of onset pertaining to the fact that family triggers an earlier incidence of diabetes.

The relationship of family history of CVD and consanguinity reveals the fact that positive family history triggers earlier onset of various cardiovascular diseases. The study showing Gujjar, Baloch and Syed having maximum percentage family history confirms the finding that these also have the majority incidences of CVD and in addition to this, their participants having an early age of onset confirms the fact that positive parental consanguinity triggers earlier cases of CVD. Furthermore, attaining second and third position among the ten other castes in terms of consanguinity, it can also be said that these caste (Gujjar and Baloch respectively) have maximum possibility of developing CVD in near future.

Lipid profile abnormalities are very common in case of heart patients and diabetics. According to the current study, raised cholesterol levels in participants belonging to Gujjar and Baloch castes reveal the fact that they also had maximum cases of cardiovascular diseases and fourth highest cases of diabetes. In addition, it can also be said that increased rate of consanguinity and strong positive family history also triggered increase in cholesterol levels and due to this; the Gujjars and Baloch had maximum proportion of the participants suffering from CVD. In contrast, Kashmiri and Rehmani had normal levels of cholesterol indicating that they had the least cases of CVD and least percentage of family history.

Followed by cholesterol levels, increased levels of triglyceride in Balochis also demonstrate the finding for why they had the maximum CVD participants. Increased cholesterol and triglyceride levels are directly linked to onset of various cardiovascular diseases as well as. According to Shaikh et al. (2010), lipid abnormalities in diabetic are mainly because of resistance to both insulin as well as hyperglycaemia and these are basically the decreased high density lipoproteins [HDL] (2b), and also increased $3 b$ and $3 c$ which are much smaller and less dense density lipoprotein (LDL= Low density lipoproteins) as well as elevated triglycerides. It was also revealed that lipid variation above normal ranges give rise to various vascular diseases, as shown by the current study as well.

Increased HDL in Rehmani and Kashmiri proves the fact for why they didn't have more cases of both CVD and diabetes. Having highest levels of HDL make them stand in a safer position and in contrast, other castes having 
lower HDL levels make them lie in the borderline. Having considerably elevated levels of LDL in Kashmiri, Gujjar and Baloch confirms the finding for why they had increased cases of diabetes and CVD.

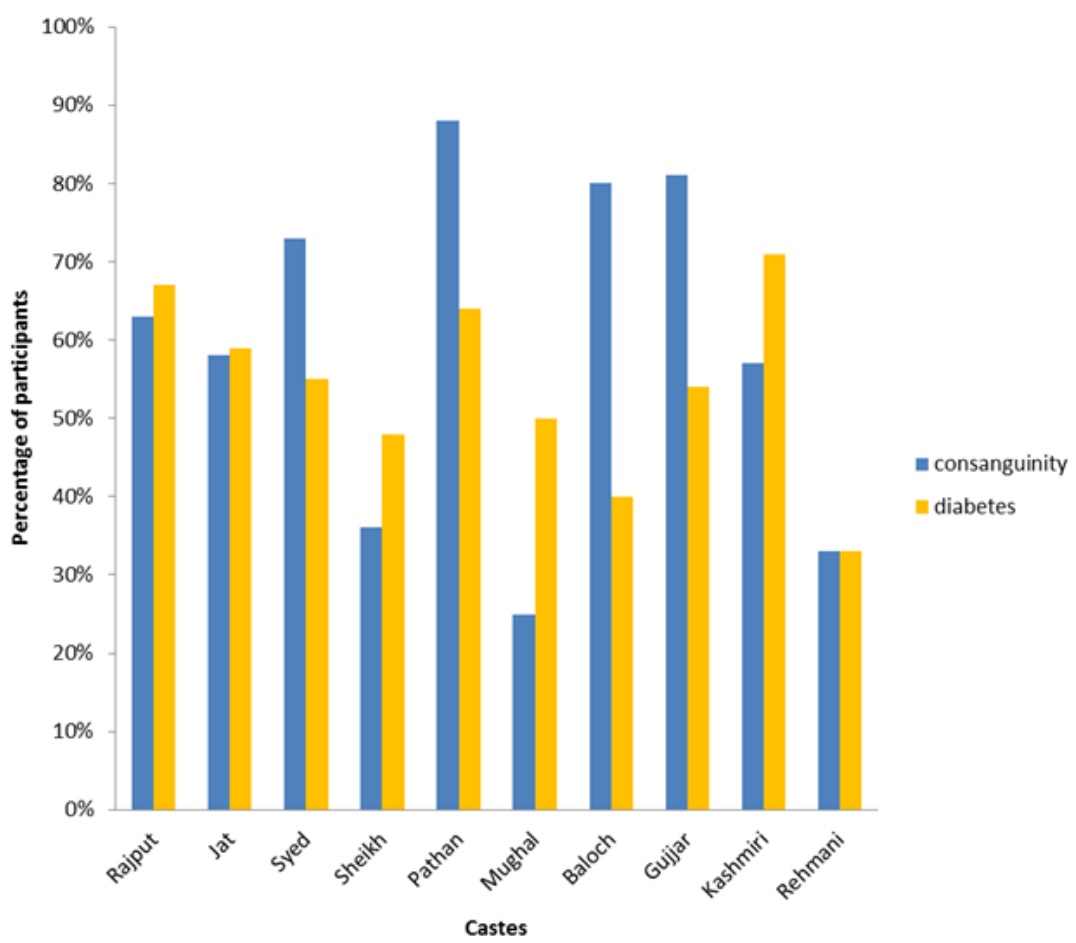

Fig I:- Relationship between consanguinity and prevalence of diabetes

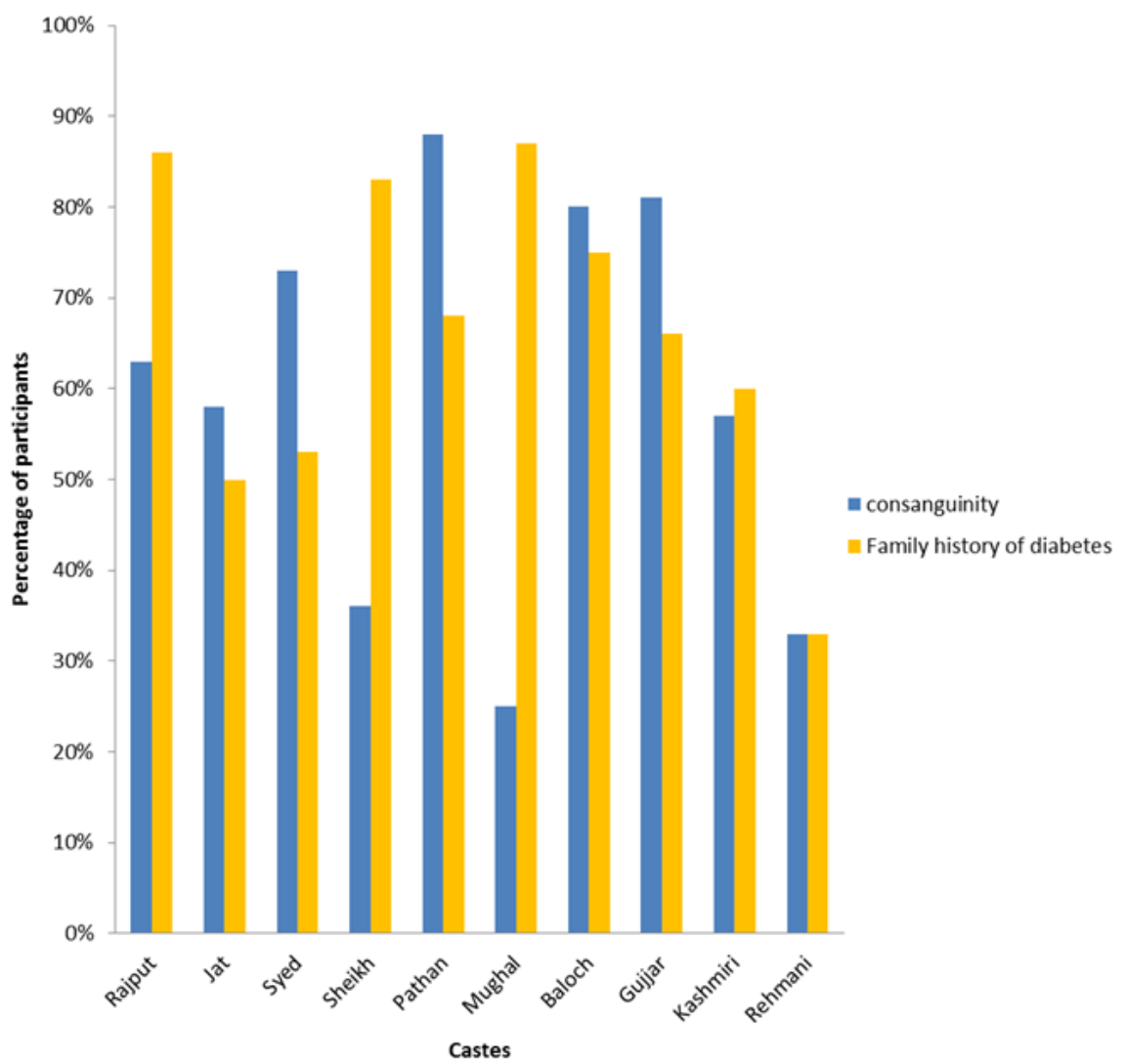

Fig II:- Consanguinity and family history of diabetes 


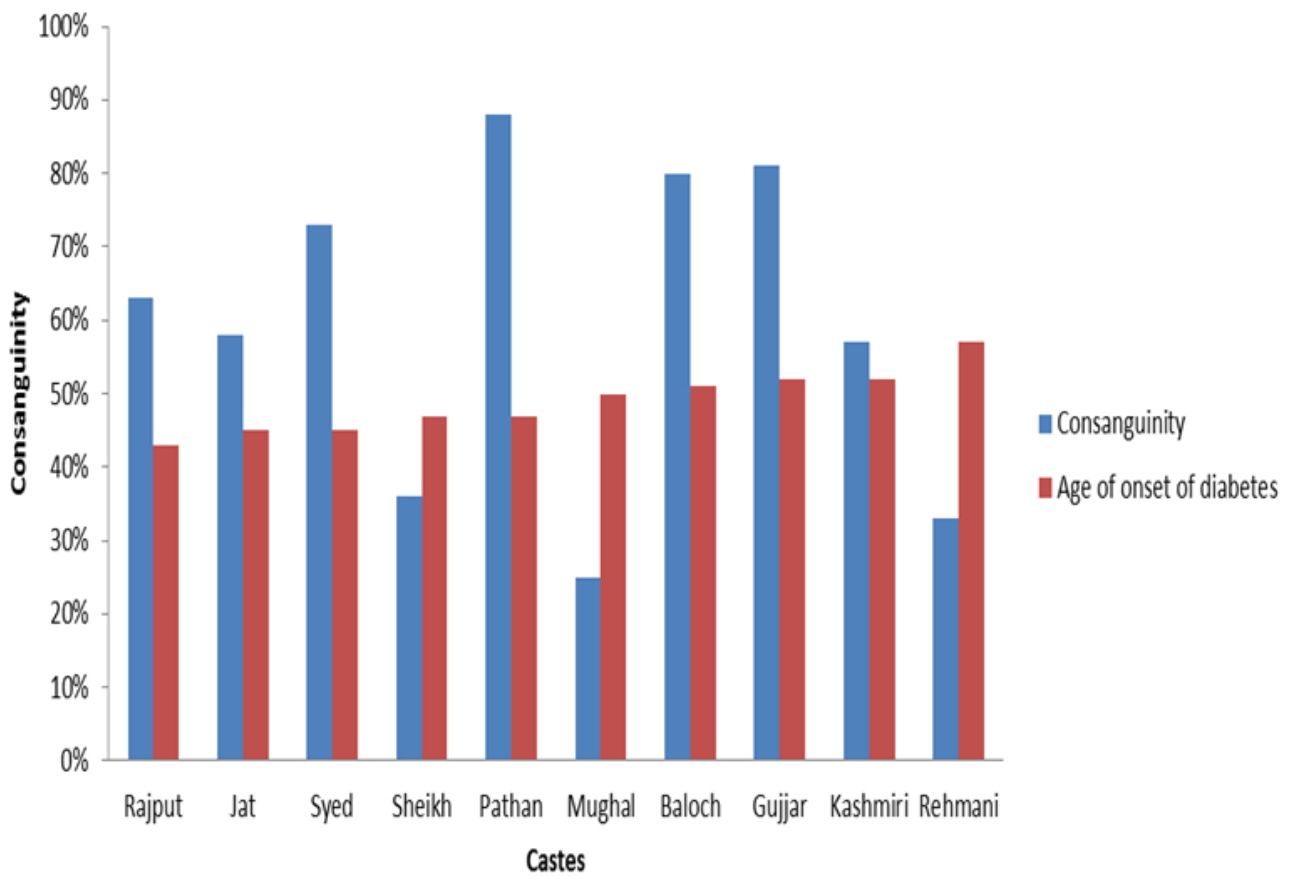

Fig III:- Age of onset for diabetes and consanguinity.

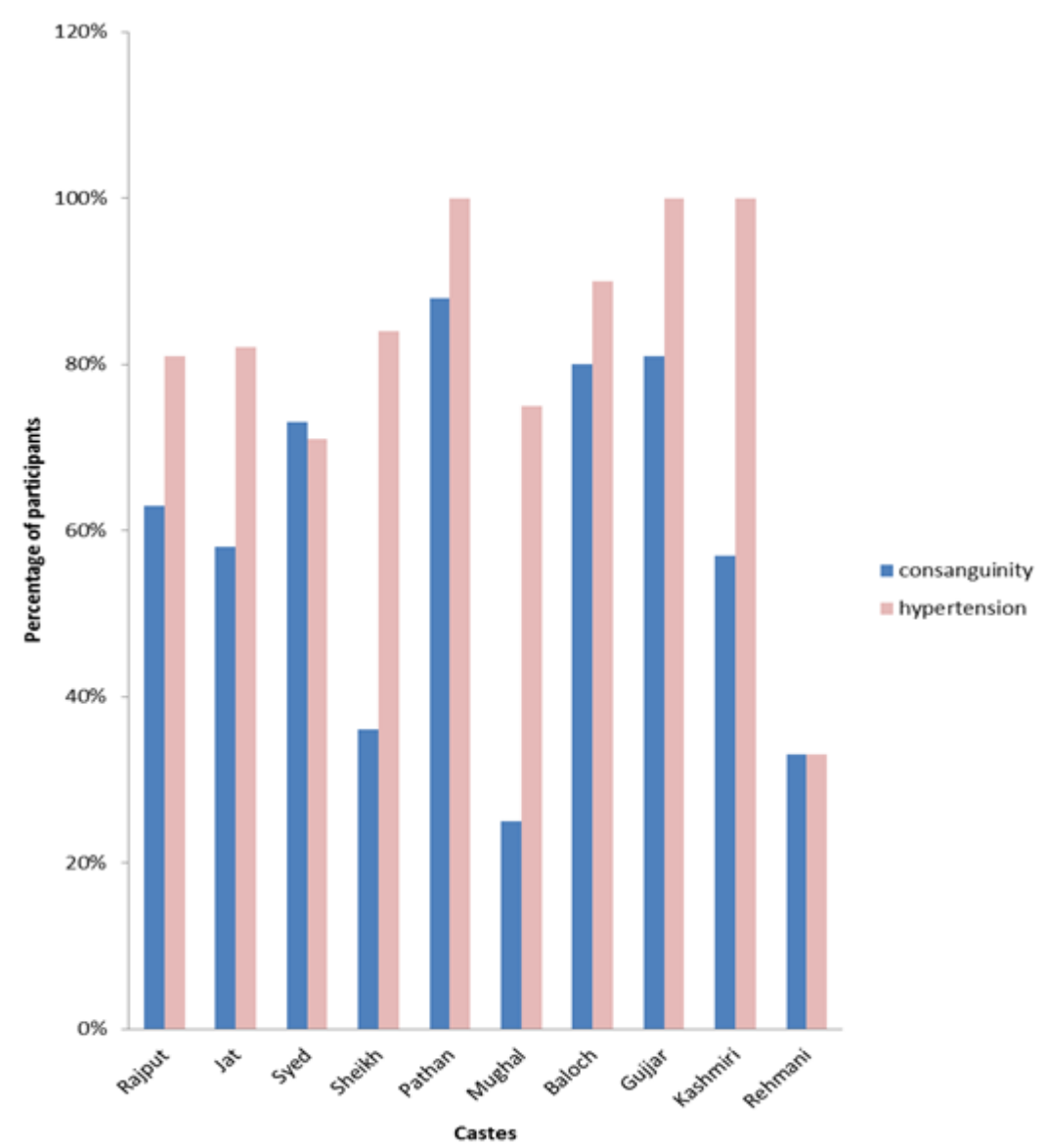

Fig IV:- Relationship between consanguinity and prevalence of hypertension. 


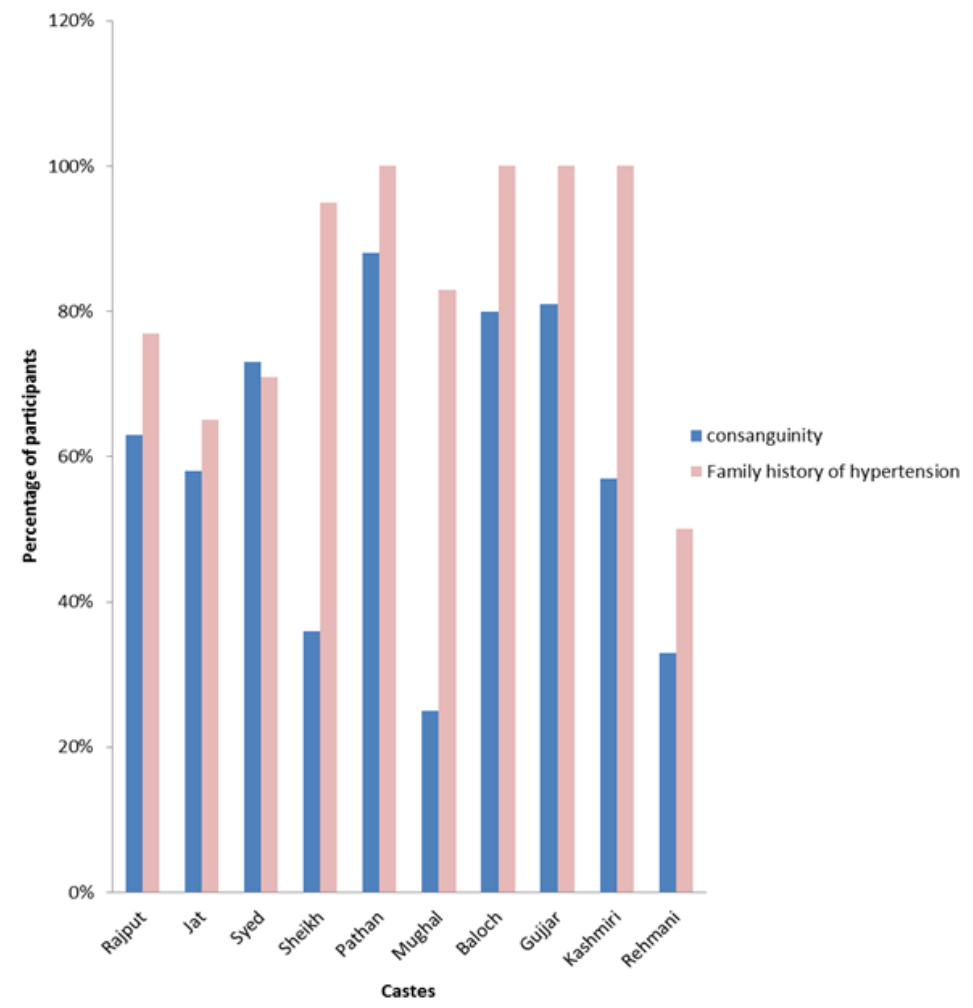

Fig V:- Consanguinity and family history of hypertension

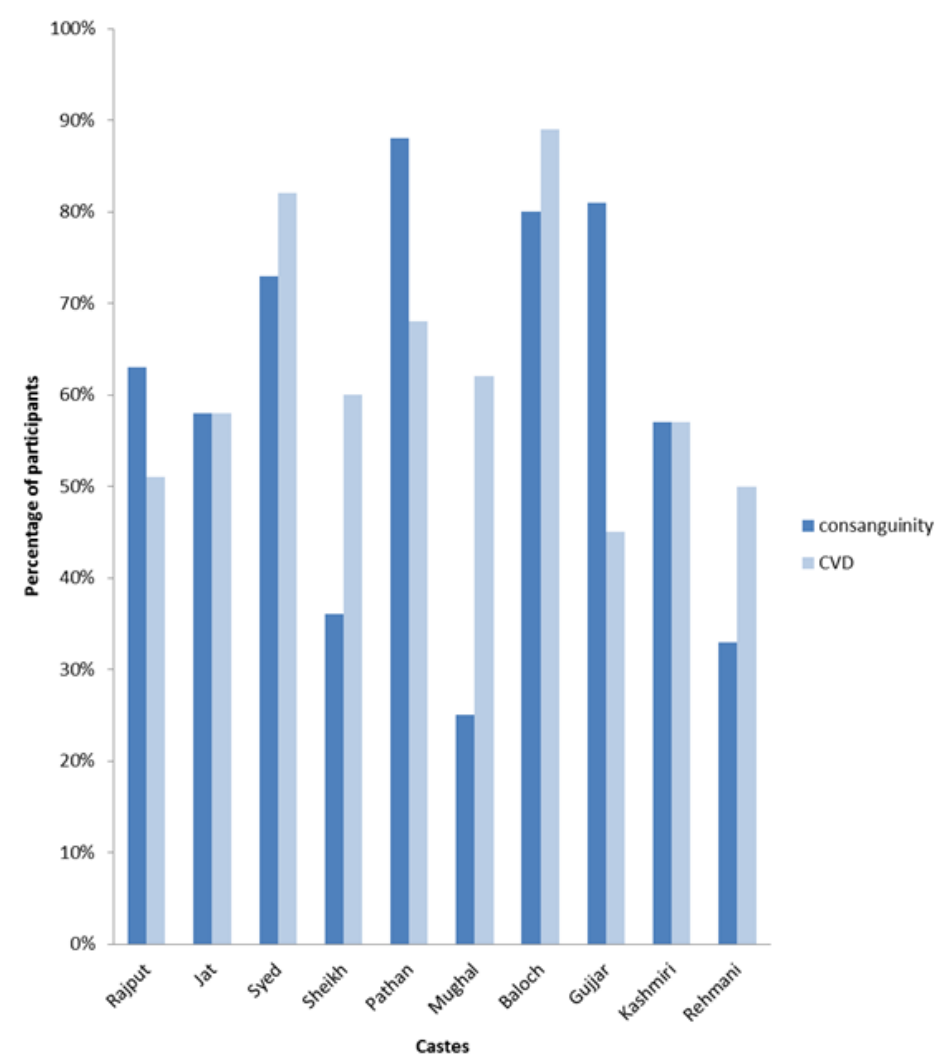

Fig VI:- Consanguinity and prevalence of cardiovascular disease 


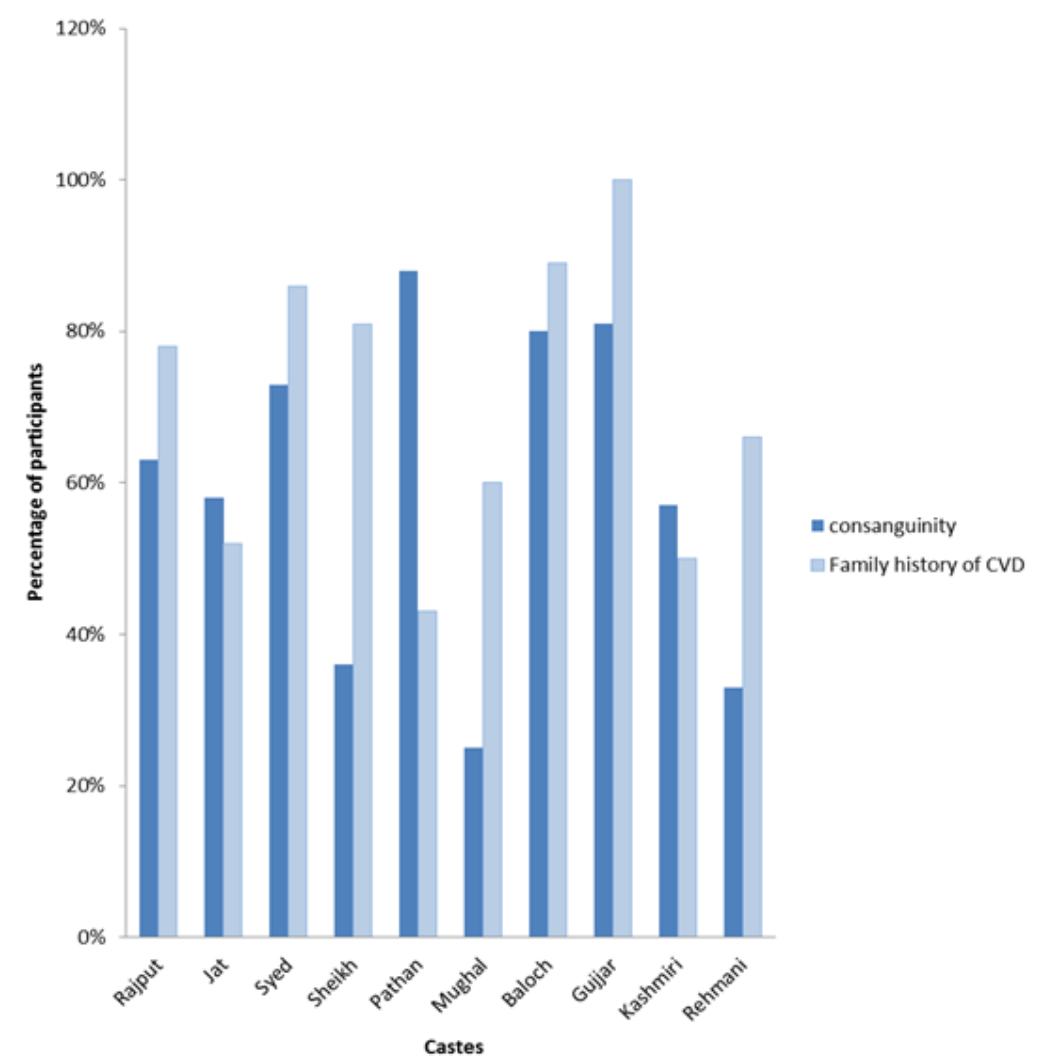

Fig VII:- Consanguinity and family history of cardiovascular disease.

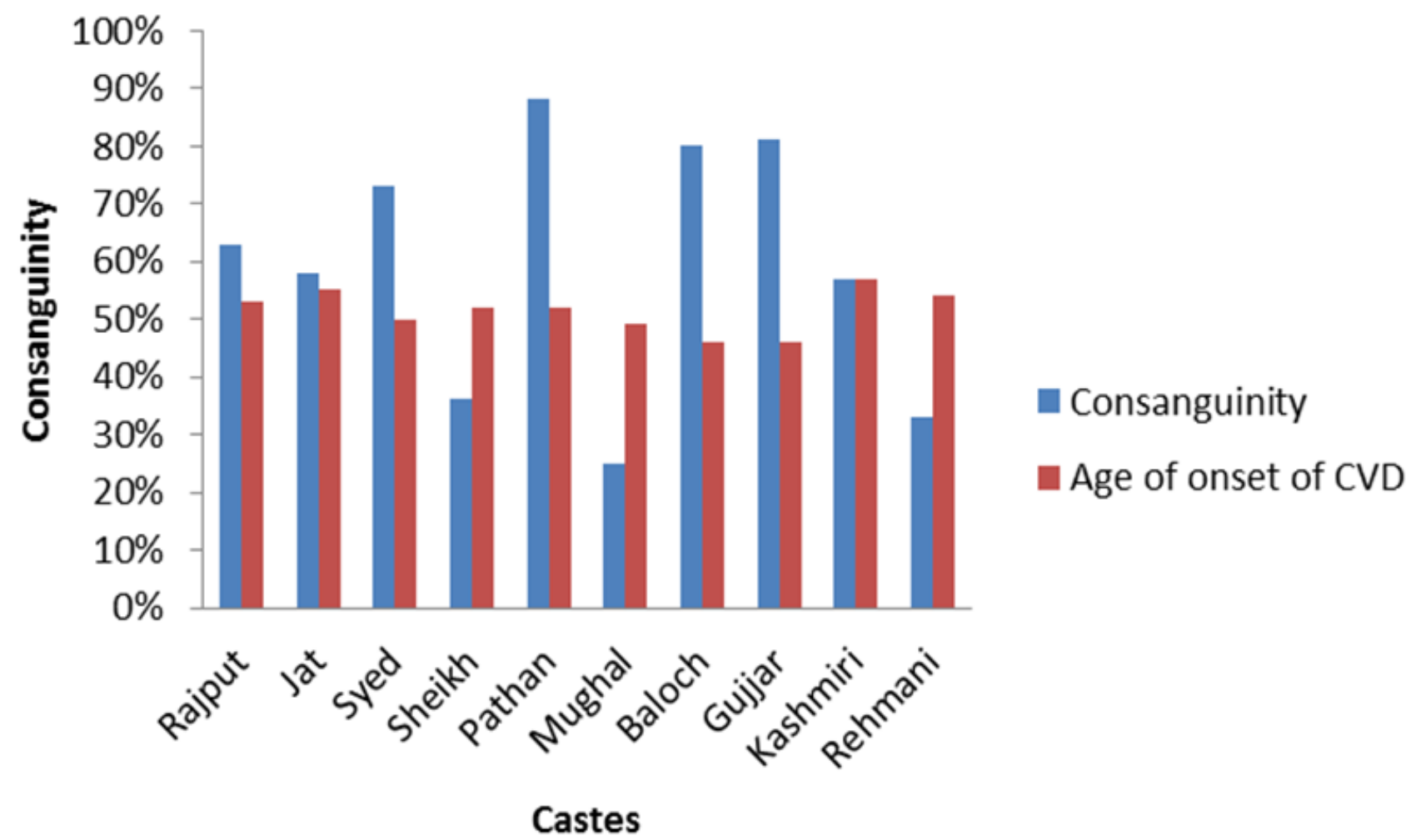

Fig VIII:- Consanguinity and age of onset for cardiovascular disease. 


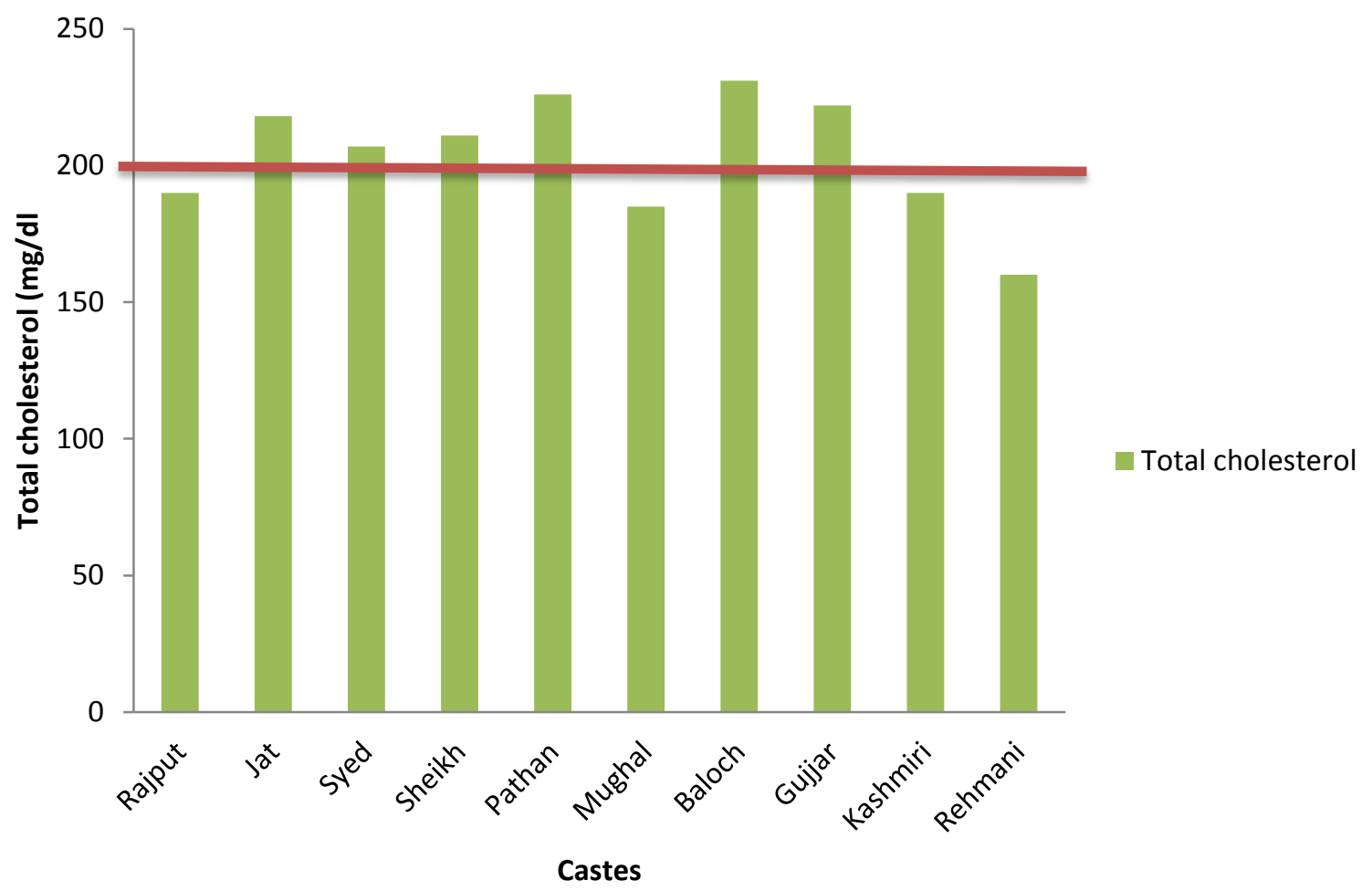

Fig IX:- Mean total cholesterol in various castes of the study.

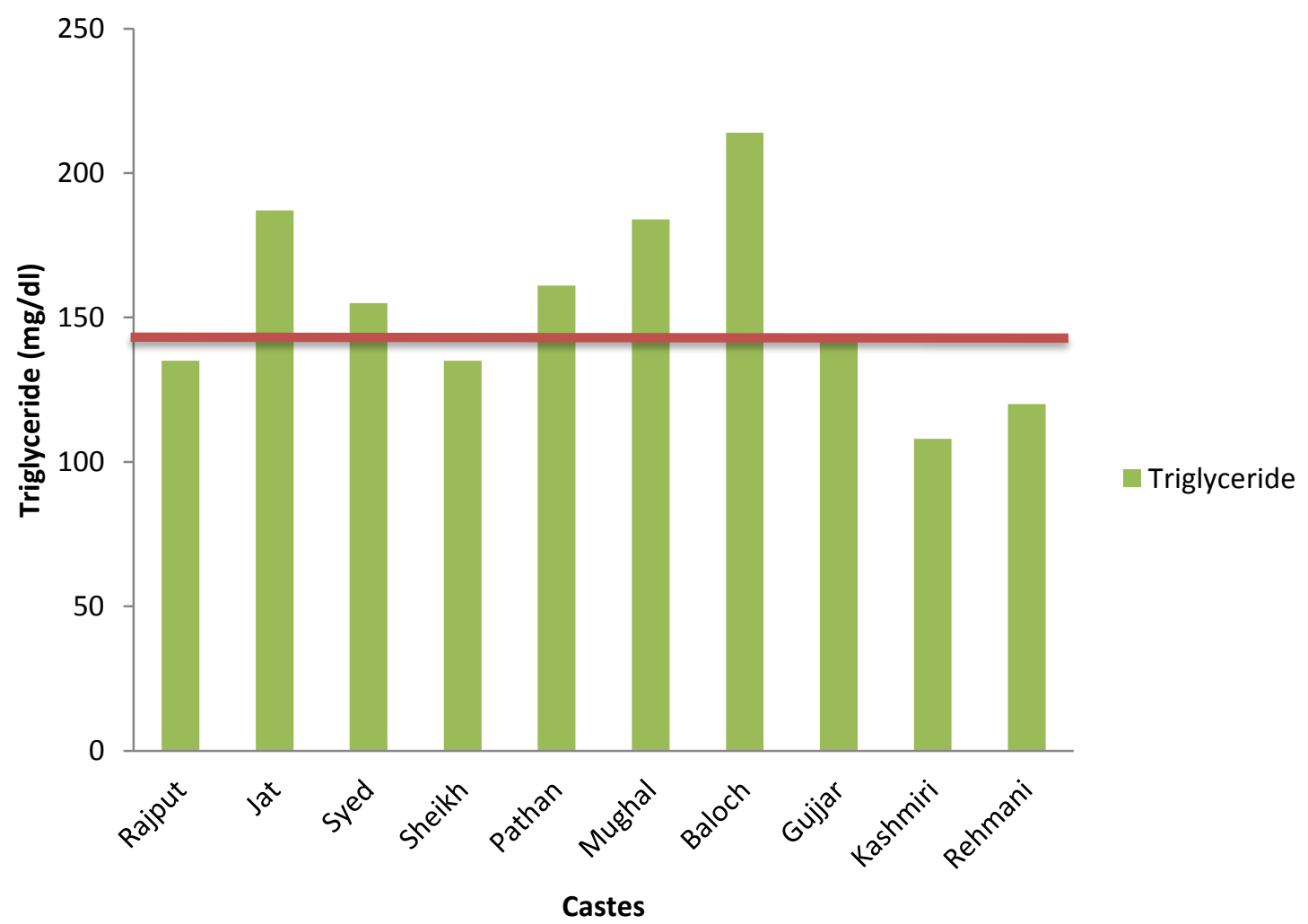

Fig X:- Mean triglyceride in various castes of the study. 


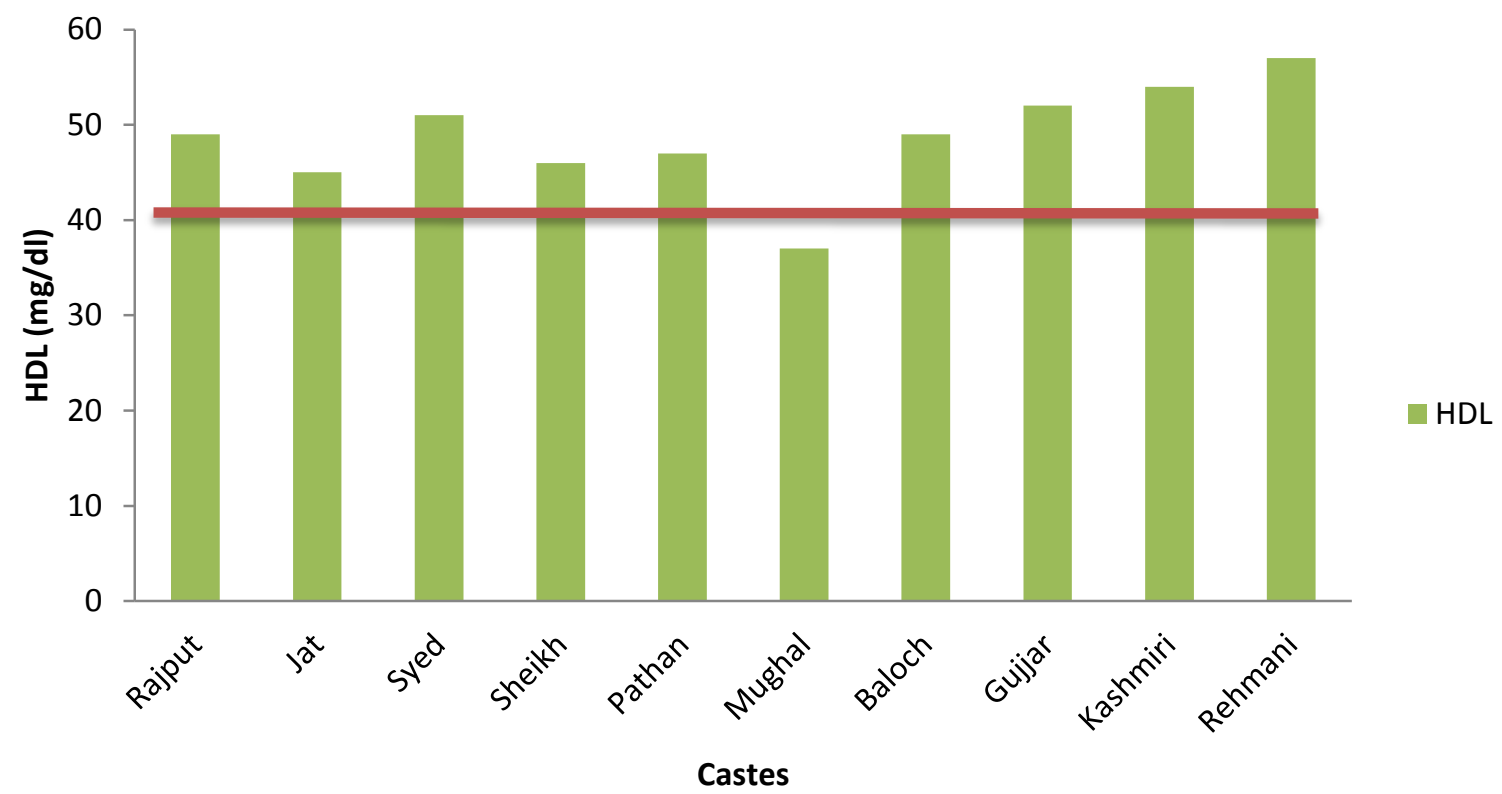

Fig XI:- Mean HDL values in males of various castes of the study.

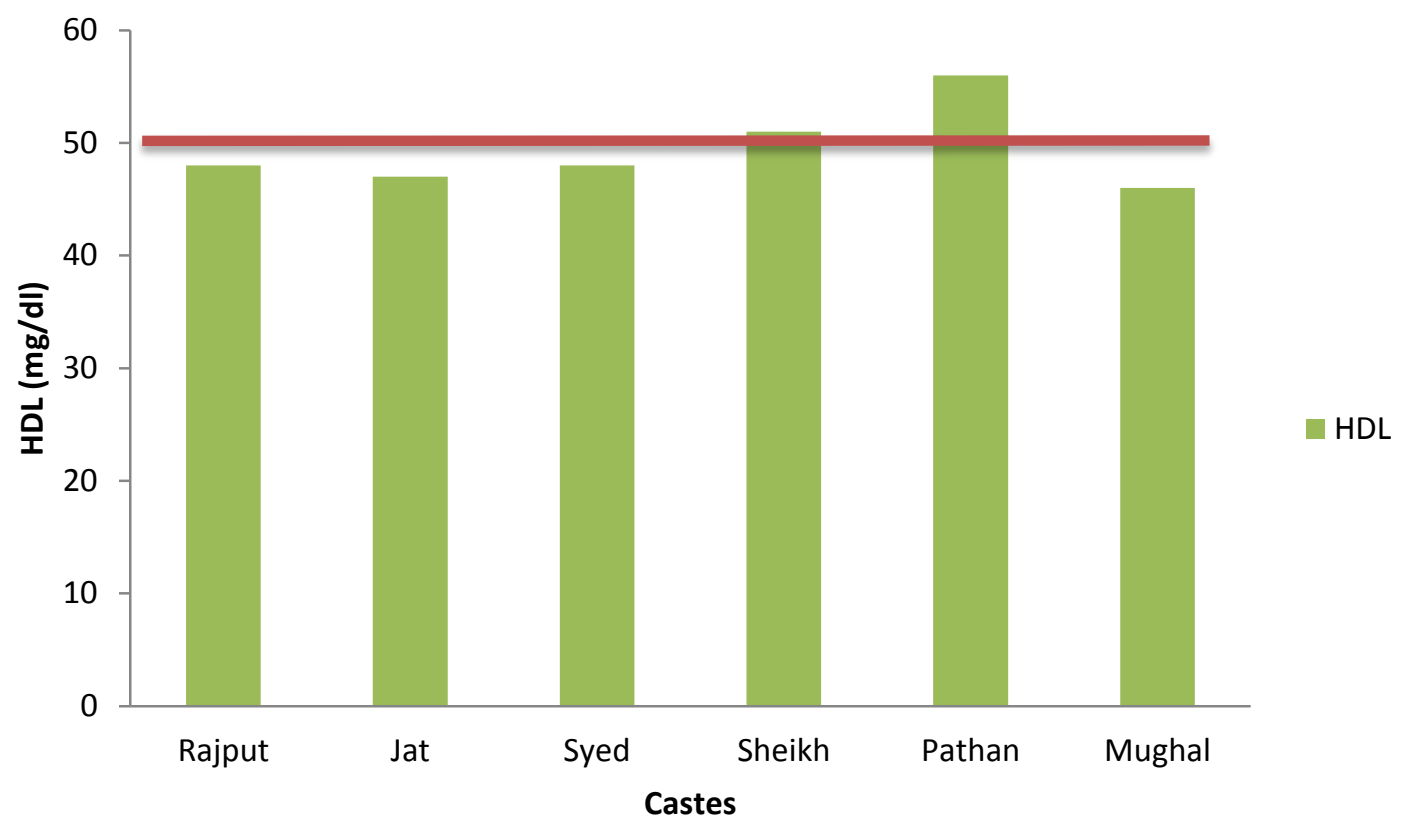

Fig XII:- Mean HDL values in females of various castes of the study. 


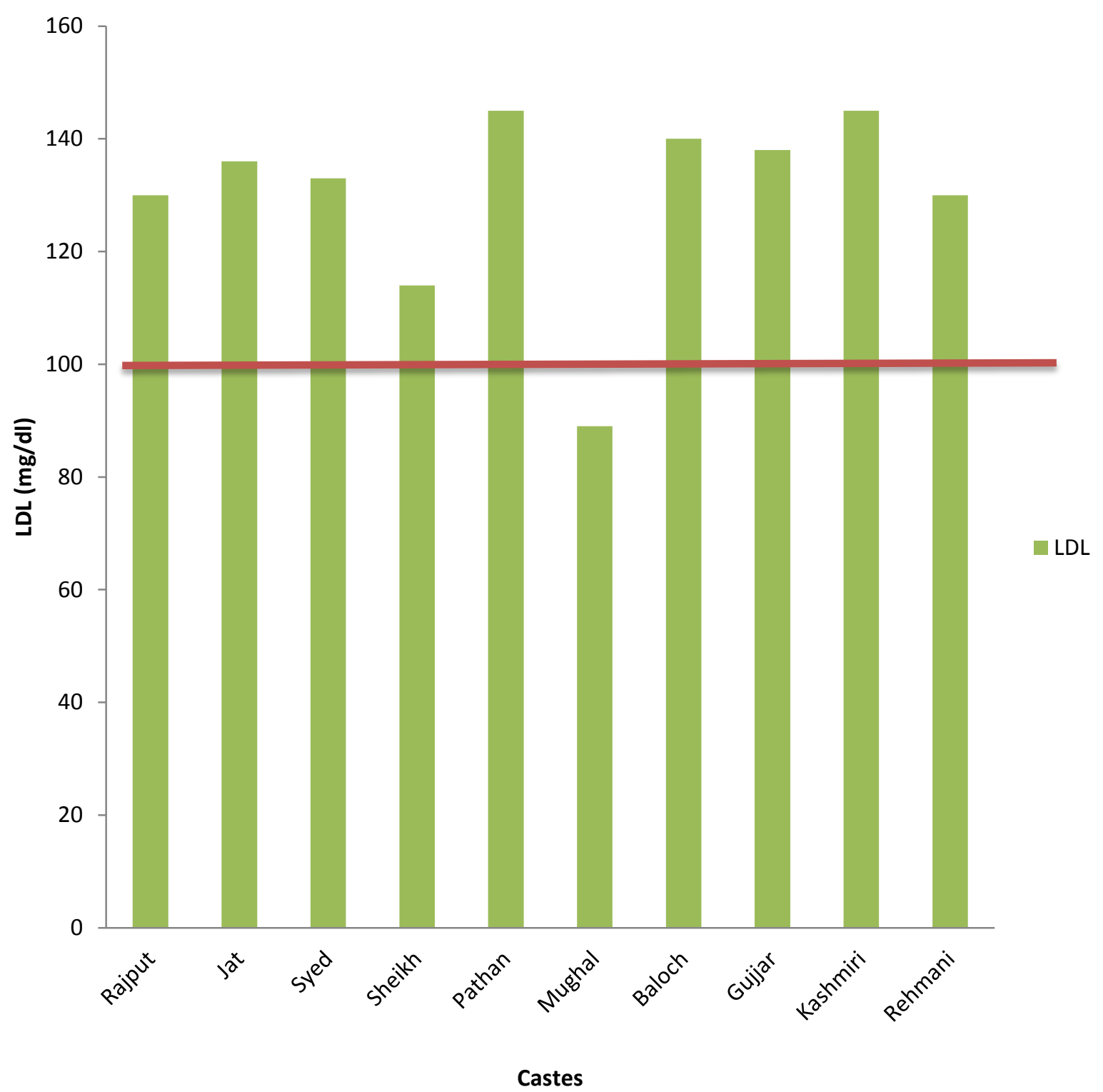

Fig XIII:- Mean LDL values in various castes of the study.

\section{Conclusion:-}

The main aim of the current study was analyzing the trends in consanguinity among various castes in Lahore which give rise to three most common complex disorders namely diabetes, cardiovascular diseases and hypertension. Results from this study show that the Pathan clan had maximum consanguinity $(88 \%)$. Furthermore, high prevalence of diabetes and hypertension in Kashmiris and cardiovascular diseases in the Baloch clan confer to the fact that consanguinity doesn't play any role in onset of these diseases but parental consanguinity may trigger the onset of these diseases in future generations. In addition Baloch and Gujjars were found to have abnormal values for various parameters of lipid profile clearly revealing presence of cardiovascular diseases among them. 


\section{References:-}

1. Ahmed, A.S. (1976): Millennium and Charisma among Pathans: A Critical Essay in Social Anthropology. United Kingdom: London: Routledge \& Kegan Paul.

2. Gupta, G.R. (1974): Marriage, Religion and Society. India: India: Gurozon Press.

3. Kaplan, N.M. (1989): The Deadly Quartet - upper-body obesity, glucose intolerance, hypertriglyceridemia, and hypertension. Arch Intern Med., 149:1514-20.

4. Kazim, S.F., Salman, M.B., Zubairi, A.J., Afzal, A., Ahmad, U. and Frossard, P.M. (2008): Offspring of Hypertensive Parents Have Higher Blood Pressure and BMI. J. Coll. Physicians Surg. Pak., 18(1): 64-65.

5. Khan, G.A., Sultana, A. and Siraj A. (2011): Consanguinal Marriages in Pakistan: A Tradition or a Cultural Politics and Possible Management Measures? Can. Soci. Sci., 7(1): 119-123.

6. Koning, L., Merchant, A.T., Pogue, J. and Anand, S.S. (2007): Waist circumference and waist-to-hip ratio as predictors of cardiovascular events: meta-regression analysis of prospective studies. Eur. Heart J., 28: 850-856.

7. Lipid Laboratory Johns Hopkins. Laboratory Procedure Manual.

8. Miller, M. (1999)" The epidemiology of triglycerides as a coronary artery disease risk factor. Clin. Cardiol., 22: 111-16.

9. Mosca, L., Barrett-Connor, E. and Wenger, N.K. (2011): Recent Advances in Preventive Cardiology and Lifestyle Medicine. Circulation., 124: 2145-2154.

10. Perveen, F. and Rehman, S. (2012): Consanguineous Marriages and the Malformation in their F1 Generation. Asia. J. Pharma. Heal. Sci., 2(3): 406-411.

11. Sabean, D.W. (1998): Kinship in Neckarhausen, 1700-1870. USA: Los Angeles: Cambridge University Press.

12. Samatha, P., Venkateswarlu, M. and Siva, P.V. (2012): Lipid Profile Levels in Type 2 Diabetes Mellitus from the Tribal Population of Adilabad in Andhra Pradesh, India. J. Clin. Diagn. Res., 6(4): 590-592.

13. Shaikh, M.A. et al. (2010): Type 2 Diabetes Mellitus and Lipid Abnormalities. JLUMHS, 9(3): 145-147.

14. Shami, S.A., Qaisar, R. and Bittles, A.H. (1991). Consanguinity and adult morbidity in Pakistan. Lance, 338: 954-955.

15. Temtamy, S. A., Meguid, A.N., Mazen, I., et al. (1998): Genetic epidemiological study of malformations at birth in Egypt. East Medit. Heal. J., 4(2): 252-259.

16. The American Diabetes Association. (1999): The management of dyslipidaemia in adults with diabetes. Diabetes Care., 22: S56-S59.

17. World Health Organization. (1994): Expert committee on prevention and treatment of diabetes mellitus. WHO technical series No 844. 